\title{
Control of a Class of Sulfate Reducing Chemostat Via Feedback Polynomial Injection
}

\author{
R. Aguilar-Lopez ${ }^{* 1}$, V. Peña-Caballero ${ }^{2}$, M. I. Neria-Gonzalez ${ }^{3}$ \\ ${ }^{1,2}$ Departamento de Biotecnología y Bioingeniería \\ CINVESTAV-IPN \\ 2508 I.P.N. Av., San Pedro Zacatenco, Mexico City, Mexico, 07360 \\ *raguilar@cinvestav.mx \\ ${ }^{3}$ División de Ingeniería Química y Bioquímica \\ Tecnológico de Estudios Superiores de Ecatepec \\ Tecnológico Av., Ecatepec, Mexico, 53210
}

\begin{abstract}
The main goal of this work is to present a new class of feedback controller which contains on its structure a polynomial form of the named control error, the proposed controller is applied to a class of sulfate-reducing chemostat in order to control the sulfate concentration, which would be useful for several biotechnological issues, as heavy metal removal in wastewater. The closed-loop behavior of the chemostat is theoretically analyzed and a practical convergence to the selected optimum trajectory is proved. The proposed methodology is applied to an experimentally corroborated kinetic model of a sulfate-reducing bacterium and further numerical experiments show the satisfactory closed-loop performance of the process in comparison with other controllers.
\end{abstract}

Keywords: Sulfate reducing chemostat, polynomial feedback, closed-loop stability.

\section{RESUMEN}

El principal objetivo de este trabajo es el presentar una nueva clase de controlador de tipo retroalimentado, el cual contiene en su estructura una forma polinomial del llamado error de control, el controlador propuesto es aplicado a un quimiostato sulfato-reductor, el cual pudiera ser usado para varios fines biotecnológicos, como la remoción de metales pesados en aguas residuales. El comportamiento a lazo cerrado del quimiostato considerado es teóricamente analizado y se prueba convergencia práctica a la trayectoria óptima seleccionada. La metodología propuesta es aplicada a un modelo cinético de una bacteria sulfato-reductora experimentalmente validado y experimentos numéricos complementarios muestran un comportamiento a lazo cerrado satisfactorio en comparación con otros controladores.

\section{Introduction}

Sulfate reducing bacteria (SRB) are dispersed in the nature, are able to produce energy through electron transfer-coupled phosphorylation, use the sulfate as the terminal electron acceptor for respiration and produce sulfide as compound end. SBR play an important role in global cycling of numerous elements in anaerobic environments, particularly in sulfate rich environments, the global sulfur and carbon cycles are most relevant (Widdel, 1988). Beyond their role in ecology, SRB have an economic impact because they are associated with pitting corrosion of ferrous metals due to production of sulfide, which is highly reactive, corrosive and toxic, particularly in the petroleum industry (Neria-Gonzalez et al.; 2006).
On other hand, SRB have become especially important in biotechnological processes due to its ability to degrade organic material and remove heavy metals (Jorgensen, 1982). Many processes of material degradation and heavy metal removal are carried on anaerobic bioreactors, which are large fermentation tanks provided with mechanical mixing, heating, gas collection, sludge addition and withdrawal ports, and supernatant outlets. The anaerobic digestion is affected by many factors including temperature, hydraulic time, $\mathrm{pH}$, and chemical composition of wastewater. In a practical framework, the bioreactors are operated on the basis of laboratory analysis of samples, which lead to empirical methodologies for operation policies. In general the above is related to the rising energy costs, increased global competition in terms of 
both price and quality, and the need to make products in an environmentally benign manner. Many of the products obtained by the biological route either cannot be produced, or are very difficult to obtain, by conventional manufacturing methods.

Generally speaking, the following features distinguish the biochemical reactors from the chemical reactors:

1. Several of the crucial variables can not be directly measured quickly or easily. Quite often a time delay, which is larger than the system timeconstants, is associated with their measurement. Therefore, a mathematical model must be used in place of feedback information.

2. As already pointed out, the fed-batch reactor operation does not have a true steady state. In this case, evaluation of the state variables will locate the position of the system on a trajectory through the operational cycle. Since these state variables can not be measured online, the estimation of state becomes an important element of optimization and control of the reactor.

3. Linear system analysis is mostly not applicable in case of bioreactors, especially for evaluation of long term response, since biochemical reaction systems usually are nonlinear. Hence, numerical solution of differential equations is required.

Biological reactors typically are governed by highly nonlinear behavior occurring on both a macroscopic reactor scale and a microscopic cellular scale. Reactions taking place at these scales also occur at different rates so that the bioreactor system is multi-scale both spatially and temporally. By increasing our understanding of these systems, it may be possible to avoid some of the empiricism associated with the operation of continuous and (fed-) batch bioreactors. Considerable benefit, in terms of reduced product variability and optimal resource utilization could be achieved. Process control mainly focuses on controlling variables such as pressure, level, flow, temperature, $\mathrm{pH}$, level in the process industries; however, the methodologies and principles are the same as in all control fields. The early successful application control strategy in process control is in evolution of the PID controller and Ziegler-Nichols tuning method (Ziegler and Nichols, 1942). So far a high percentage of the controllers implemented in the process industries are PID-type (Chidambaram and See, 2002); however, as (i) the industrial demands (ii) the computational capabilities of controllers and (iii) complexity of systems under control increase, the challenge is to implement advanced control algorithms (Spear, 2005).

Since achievable controller performance in a model-based control scheme is dependent on the quality of the process model (Aguilar, et al, 2005), a controller based on a model that captures events occurring at both the general considerations for control of bioreactors.

On the other hand, the difficulty of implementing a feedback control is threefold. Firstly, the response of sensors tends to be slower than many of the processes they monitor. Secondly, sensors are generally not available for measurement of substrate with rapid dynamics for feedback application; and thirdly, there is also generally not available a sensor for the biomass concentration without which the state of the system can not be estimated (Fordyce, et al, 1987)

Given the above objectives, broadly speaking, there are two ways to design an appropriate control system. The most frequently used method is to pre-select a controller structure and then to tune the parameters of this controller so that the desired closed-loop response is obtained. This is referred to as a parameter optimized control system; the most well-known example is probably the Proportional Integral Derivative (PID) controller (Bastin and Dochain, 1990). The other approach is the use of structure optimal control systems where both the structure and parameters of the controller are adapted to those of the process model (Betancour, et al, 2006). In practice, however, the use of the latter method is severely restricted because exact dynamic term cancellation is required in order to produce the optimal controller structure. This is usually not possible for various reasons: the lack of an appropriate process model, non-linearities and physical constraints on the process variables.

From the above, in this paper a class of nonlinear single-input single-output controller with polynomial output structure in order to provide stabilization to a class of continuous sulfate reducing bioreactor is proposed. 


\section{Experimental}

In this paper, a sulfate-reducing bacterium (6SR), isolated from a biofilm sample obtained from the inner surface of pipelines that transport oil crude, is considered. The molecular identification of $6 \mathrm{SR}$ isolated showed a phylogenetic relation with Desulfovibrio alaskensis so the strain is named Desulfovibrio alaskensis 6SR (Neria-Gonzalez et al.; 2006). Desulfovibrio alaskensis 6SR was cultured and conserved in Ravot medium under an atmosphere of N2-CO2 (80:20, v/v).

\subsection{Growth kinetic}

A sample of congenital water medium (CW) was obtained from an oil pipeline located in the Mexican Southeast region. Chemical determination of water: chlorides $64000 \mathrm{ppm}$, sulfur $178 \mathrm{ppm}$, sulfate 350 to $400 \mathrm{ppm}, \mathrm{pH} 8.84$. A $1000 \mathrm{~mL}$ aliquot of congenital water was saturated whit N2 for an hour and was enriched with sodium lactate 6 $\mathrm{mL}$, yeast extract $0.5 \mathrm{~g}$, and reducing solution 5 $\mathrm{mL}$ (acid ascorbic $1 \mathrm{~g} \mathrm{~L}^{-1}$, and sodium thioglicolate, $\left.1 \mathrm{~g} \mathrm{~L}^{-1}\right)$. The $\mathrm{pH}$ was adjusted to 7 with $\mathrm{KOH} 1 \mathrm{~N}$. The CW medium was distributed in serum bottles of $60 \mathrm{~mL}$ usingthe Hungate's technique (Hungate, 1969) and they were autoclaved at $120^{\circ} \mathrm{C}$ for 15 minutes.

The original cultures of D. alaskensis $6 \mathrm{SR}$ in medium Ravot were used to inoculate $45 \mathrm{~mL}$ of $\mathrm{CW}$ medium. The culture was incubated for 20 days at $37^{\circ} \mathrm{C}$. This was used to inoculate three bottles with $\mathrm{CW}$ medium for different periods of time: zero, 24 and 36 hours, respectively, and were incubated under same conditions. The bacterial growth was followed through Optical Density (OD) measurements, consume of sulfate and production of sulfide. Samples from the cultures were taken anaerobically each hour. Sulfate in the medium was measured by the turbidimetric method based on the precipitation of barium (Kolmert $\AA$ et al., 2000). Also, the production of sulfide was measured by a turbimetric method (Cord-Ruwisch, 1985). The OD reading for cell growth was transformed to dry weight $(\mathrm{mg} / \mathrm{mL})$ through a standard curve of growth. The data were analyzed and only the points that adjusted a straight line (exponential phase) were used to determine the growth kinetic parameters according to the Monod model.

\section{Process modeling}

Sulfate reduction can be applied beneficially to biotechnology, i.e. for the the removal of heavy metals from groundwater and waste water. These applications take advantage of differences in the chemical properties of metal sulfates and sulphides. Metal sulfates (cadmium, cobalt, copper, iron, nickel and zinc) are highly soluble but the corresponding metal sulphides have low solubility. Thus, by sulfate reduction, metals can be precipitated, recovered and reused. This concept has been applied to immobilize metals from surface water and process water from mining industries. Organic waste materials (for example, straw) are often used to immobilize heavy metals in lake sediments. From the above, the necessity to improve the operating performance of these processes seems very important and the system modeling, simulation, optimization and control appear to be tools for a complete process analysis.

For biological systems, the unstructured kinetic models are the simplest of all modeling philosophies used to describe the biological model. They consider the cell mass as a single chemical species and do not consider any intracellular reactions occurring within the cell. Unstructured models typically describe the growth phenomena based on a single limiting substrate and consider only substrate uptake, biomass growth, and product formation in the modeling framework. Thus the biological component of the system depends directly on the macroscopic reactor variables. These models give an adequate representation of the biological growth phenomena in relatively simple cases, when the cell response time to environmental changes is either negligibly small or much longer than the batch time (Schugerl, 1987).

The most commonly used unstructured model in the literature is the Monod model (Alford, 2006). And it is one of the earliest attempts at modeling biological systems. In this model the growth kinetics are expressed in terms of the specific growth rate. The parameters are obtained experimentally and they do not have a direct physical interpretation (Schugerl, 1987). A drawback of this model is that it does not capture the initial lag phase of growth which is observed in most batch cultures. There are variants to the Monod model that have also been used, such as 
the models by Messier, Contois etc. reported in the open literature (Bailey and Ollis, 1986). These models differ in their substrate dependence and some include terms to account for saturation due to high substrate concentration and inhibition due to product or a competing inhibitor; however, these models do not differ significantly from the Monod model in the fact that they are empirical and represent all of the cellular processes with just a single equation for the specific growth rate.

Nevertheless, for control purposes, a reduced order model which can describe the dynamic behavior of the main state variables is adequate. The kinetic parameters can be determinated via standard methodology (Bailey and Ollis, 1986) in a batch culture (Neria-González and Aguilar-Lopez, 2007).

By employing a Monod model for the specific growth rate, the following is obtained:

$$
\begin{gathered}
\mu(S)=\mu_{\max } \frac{S}{K_{S}+S} \\
\text { or } \\
\frac{1}{\mu}=\frac{1}{\mu_{\max }}+\frac{\mathrm{K}_{\mathrm{S}}}{\mu_{\max } S}
\end{gathered}
$$

The corresponding kinetic parameters can be fitted by plotting $\mu^{-1}$ versus $S^{-1}$ (Lineweaver-Burk plot). For our particular study case, the maximum specific growth rate is calculated as $\mu_{\text {max }}=0.035 h^{-1}$ and the affinity constant $k_{s}=0.9 \mathrm{~g} / \mathrm{L}$ with a correlation factor of $\mathrm{R}^{2}=$ 0.9786 .

Therefore:

$$
\mu(S)=\frac{0.035 S}{0.9+S}
$$

and $Y_{S / X}=0.25$ and $Y_{P X X}=0.263$

The following mathematical model for the continuous bioreactor is hence proposed, based on classical mass balances for biomass, sulfate (substrate) and sulfide (product) concentrations:

Sulfate (S).-

$$
\frac{d S}{d t}=D\left(S_{i n}-S\right)-\mu(s) \frac{X}{Y_{s / X}}
$$

Biomass (X).-

$$
\frac{d X}{d t}=-D X+\mu(S) X
$$

Sulfide $(P)$--

$$
\frac{d P}{d t}=-D P+\mu(S) \frac{X}{Y_{P / X}}
$$

Here $D$ is the dilution rate (control input), $\mu$ is the specific growth rate, $Y_{S / X}$ is the sulfate coefficient yield and $Y_{P X}$ is the sulfide coefficient yield.

In a previous paper (Neria-González and AguilarLópez, 2007), some operating regions related with specific process applications (for example, heavy metal removal) were determined. From the above, two open-loop steady states were selected as mentioned below; the first equilibrium point for heavy metal remotion regimen is $S_{\text {eq }}=2.25 \mathrm{~g} / \mathrm{L}$; $X e q=0.68 \mathrm{~g} / \mathrm{L} ; P_{e q}=2.61 \mathrm{~g} / \mathrm{L}$. The second equilibrium point: $S e q=0.36 \mathrm{~g} / \mathrm{L} ; X_{e q}=1.16 \mathrm{~g} / \mathrm{L}$; Peq $=4.4 \mathrm{~g} / \mathrm{L}$, is related to high sulfate consumption.

Now, evaluation of the the stability conditions of the above equilibrium points is necessary, this is done via the first Lyapunov criteria, where the corresponding eigenvalues for the first equilibrium point are $\lambda_{1}=-1 / 40 ; \lambda_{2}=-1 / 40 ; \lambda_{3}=43 / 280$, note that the real part of the eigenvalue corresponding to sulfide concentrations is positive; therefore, the equilibrium point is unstable.

On the other side, the corresponding eigenvalues for the second equilibrium point are $\lambda_{1}=-1 / 100 ; \lambda_{2}=-1 / 100 ; \lambda_{3}=59 / 350 ; \quad$ as above, the the equilibrium point is unstable too. 
From the above results, it can be concluded that the open-loop operation is locally unstable in such a way that any disturbance arriving to the bioreactor can lead to the process to other, possibly undesirable, operating point; this justifies the application of a control law to force the bioreactor to remain on the selected operating points.

\section{Proposed controller}

A general model of the bioreactor can be described by Equations (7a) and (7b), which is related to an affine control law system for the considered process, as follows:

$$
\begin{aligned}
& \dot{X}=f(X, U)=\mathfrak{I}(X)+\ell(X) U \\
& Y=h(X)=c X
\end{aligned}
$$

Where:

$X=[S, X, P]^{T} \in \mathfrak{R}_{+}^{3}$ is the corresponding state vector. Now consider the set $\Phi \subset \mathfrak{R}^{3}$ as the corresponding physically realizable domain such that $\Phi=\left\{(S, X, P) \in \mathfrak{R}_{+}^{3} / 0 \leq S \leq S_{\text {in }} ; 0 \leq X \leq X_{\text {max } ;} ; 0 \leq P \leq P_{\text {max }}\right\}$ $; \Im(X) \in C^{\infty}(X)$, i.e. this is a smooth function; $\Im(0)=0$ and $\mathfrak{I}(X) \leq \Gamma \quad \forall X \in \mathfrak{R}_{+}^{3}$, where $\Gamma<\infty$, this means that is bounded.

Defining the vector error as $e=X-X_{s p}$, where $X_{s p}$ is the considered reference trajectory; therefore, $\dot{e}=\dot{X}-\dot{X}_{s p}$; where it is assumed that $\dot{X}_{s p}=\mathfrak{\Im}\left(X_{s p}\right)$ ; this means that the proposed trajectory (sulfate concentration) obeys the mass conservation principle despite the corresponding mass balance equation under the action of the considered control law. From the above, the corresponding error dynamic equation considering (7a) is as follows:

$$
\dot{e}=\mathfrak{I}(x)-\mathfrak{I}\left(x_{\text {sp }}\right)+\ell(x) \cup
$$

Now, let us consider the following hypothesis:

H1. For the realized control input vector $\mathbf{U}(X(t))$, $\left(\|\mathbf{U}(X(t))\| \leq \overline{\mathbf{U}}_{\text {max }}\right)$, the nominal closed-loop nonlinear system is quadratically stable; therefore, there exists a Lyapunov function $\Psi \geq 0$ that satisfies the following:

where $\Delta \mathfrak{I}(\circ)=\mathfrak{I}(X)-\mathfrak{I}\left(X_{s p}\right)$, such that the uncontrolled states of Equation (7) are stable on the quadratic domain given by (9).

H2. The nonlinear vector function $\Delta \mathfrak{I}(0)$ is Lipchitz bounded i. e.: $|\Delta \Im(\circ)| \leq \Lambda|e|$.

H3. It is considered that $|\ell(X)| \leq \ell^{+}$, where $\ell^{+}$is an upper bound.

Proposition 1. For system (7) the following control $\mathrm{U}$ is considered:

$$
U=g_{1} C\left(x-x_{s p}\right)+g_{2} C^{w}\left(x-x_{s p}\right)^{w}
$$

then $U$ is able to provide semi-global stabilization to system (7).

\section{Sketch of proof of Proposition 1.}

Taking into account the above assumptions and applying the triangle inequality, the following expression can be obtained:

$$
\begin{aligned}
& \frac{\partial \Psi}{\partial \mathrm{x}}[\Delta \mathfrak{I}(\circ)+\ell(\mathrm{x}) \mathrm{U}] \leq-\varphi_{1}\|\mathrm{x}\|^{2}, \quad\left\|\frac{\partial \Psi}{\partial \mathrm{x}}\right\| \leq \varphi_{2}\|\mathrm{X}\|, \quad \varphi_{1}, \varphi_{2}>0 \\
& |\vec{e}| \leq \Lambda|e|+g_{1} C \ell^{+}|e|+g_{2} C^{w} \ell^{+}\left|e^{w}\right|=\left(\Lambda+g_{1} C \ell^{+}\right)|e|+g_{2} C^{w} \ell^{+}\left|e^{w}\right|
\end{aligned}
$$




$$
\operatorname{sign}\left(\dot{e}_{i}\right) \dot{e}_{i} \leq\left(\Lambda+g_{1} C \ell^{+}\right)_{i} \operatorname{sign}\left(e_{i}\right) e_{i}+\left(g_{2} C^{w} \ell^{+}\right)_{i} \operatorname{sign}\left(e_{i}{ }^{w}\right) e_{i}{ }^{w}
$$

Considering a scalar element $\mathrm{i}$ of the above vector differential inequality and employing the equality: $|e|=\operatorname{sign}(e) e$, the following equation is obtained:

Therefore, after algebraic manipulations, the following differential inequality is generated:

$$
\dot{\mathrm{e}}_{\mathrm{i}}-\lambda_{1 \mathrm{i}} \mathrm{e}_{\mathrm{i}} \leq \lambda_{2 \mathrm{i}} \mathrm{e}_{\mathrm{i}}{ }^{\mathrm{w}}
$$

Where:

$$
\begin{array}{r}
\lambda_{1 \mathrm{i}}=\frac{\left(\Lambda+\mathrm{g}_{1} \mathrm{C} \ell^{+}\right)_{\mathrm{i}} \operatorname{sign}\left(\mathrm{e}_{\mathrm{i}}\right)}{\operatorname{sign}\left(\dot{\mathrm{e}}_{\mathrm{i}}\right)} \\
\text { and } \lambda_{2 \mathrm{i}}=\frac{\left(\mathrm{g}_{2} \mathrm{C}^{\mathrm{w}} \ell^{+}\right)_{\mathrm{i}} \operatorname{sign}\left(\mathrm{e}_{\mathrm{i}}^{\mathrm{w}}\right)}{\operatorname{sign}\left(\dot{\mathrm{e}}_{\mathrm{i}}\right)}
\end{array}
$$

To solve the above inequality, consider the change of variable:

$$
\vartheta_{\mathrm{i}}=\mathrm{e}_{\mathrm{i}}^{1-\mathrm{w}}, \mathrm{w}>1
$$

Thus

if $e_{i}=0 \Rightarrow \vartheta_{i}=0$

and

if $e_{i} \neq 0 \Rightarrow \vartheta_{i} \neq 0$, i.e.

$\left\{\begin{array}{l}e_{i}>0 \\ e_{i}<0, w \text { odd }\end{array}\right\} \Rightarrow \vartheta_{i}>0$

Here after we consider

$e_{i} \neq 0 \quad$ with $\quad w \in Z^{+}, w$ odd,$w>1$.
Considering that inequality (12) is a Bernoulli type differential inequality and taking into account the change of variable (15), the next first order ordinary differential inequality is generated:

$$
\dot{\vartheta}_{i}-(1-w) \lambda_{1 i} \vartheta_{i} \geq(1-w) \lambda_{2 i}
$$

By solving the above:

$$
\begin{aligned}
& \vartheta_{\mathrm{i}} \geq \vartheta_{0 \mathrm{i}} \exp \left(-\{\mathrm{w}-1\} \lambda_{1 \mathrm{i}} \mathrm{t}\right)+\frac{\lambda_{2 \mathrm{i}}}{\lambda_{1 \mathrm{i}}}\left(1-\exp \left(-\{\mathrm{w}-1\} \lambda_{1 \mathrm{i}} \mathrm{t}\right)\right) \\
& \text { For } t \rightarrow t_{\infty} \\
& \qquad \vartheta_{\mathrm{i}} \geq \frac{\lambda_{2 \mathrm{i}}}{\lambda_{1 \mathrm{i}}}
\end{aligned}
$$

To get back to the original variable (regulation error):

$e_{i} \leq\left(\frac{\lambda_{1 i}}{\lambda_{2 i}}\right)^{\frac{1}{w-1}}=\left(\frac{\left(\Lambda+g_{1} C \ell^{+}\right)_{i} \operatorname{sign}\left(e_{i}\right)}{\left(g_{2} C^{w} \ell^{+}\right)_{i} \operatorname{sign}\left(e_{i}^{q}\right)}\right)^{\frac{1}{w-1}}, w>1$

Defining the maximum error as $\bar{e}=\max _{i} e_{i}$. it can be concluded that $\bar{e} \in B_{\bar{\varepsilon}}(0) .2$

\section{Remarks:}

Note that the estimation error can be diminished as desired, considering $g_{2}$ large enough or considering $g_{1} \approx-\Lambda\left(C \ell^{+}\right)_{i}^{-1}$, both considerations can be used as tuning rules guidelines; besides, as $w$ increases, the regulation error is diminished as shown in Equation (20). 


\section{Numerical experiments and results}

Firstly, the performance of the experimental kinetic model is shown in Figure 1, where an adequate performance can be observed. On the other hand, several numerical simulations were carried out in order to show the performance of the proposed methodology. The mathematical model of the considered continuous bioreactor was simulated via Math Lab tools (23S ODE library) ®; the corresponding initial conditions for the sulfate, biomass and sulfide concentrations are $6.0 \mathrm{~g} \mathrm{~L}^{-1}$, $1.0 \mathrm{~g} \mathrm{~L}^{-1}$ and $0.1 \mathrm{~g} \mathrm{~L}^{-1}$, respectively. The bioreactor is operated in an open-loop mode from the zero time at $50 \mathrm{~h}$ when the controller is tuning up, the original set point is defined at $2.25 \mathrm{~g} \mathrm{~L}^{-1}$; at $\mathrm{t}=75$ $\mathrm{h}$, the set point changes to $0.36 \mathrm{~g} \mathrm{~L}^{-1}$ and, finally, at $\mathrm{t}=125 \mathrm{~h}$, the set point is $1.5 \mathrm{~g} \mathrm{~L}^{-1}$ of sulfate. For comparison purposes, several controllers were implemented; an ideal Input/Output linearizing controller (Kalafatis, et al, 2005), linear PI controller and the proposed methodology. The linear PI controller was tuned by Internal Model Control (IMC) guidelines (Rivera et al, 1986); the corresponding tuning is done via a step disturbance of $5 \%$ in the nominal value of the control input $\left(D=0.025 \mathrm{~h}^{-1}\right)$, the steady-state gain is calculated as $\mathrm{K}=1400 \mathrm{~g} \mathrm{~h} \mathrm{~L}^{-1}$, the characteristic time is $\tau=170 \mathrm{~h}$, the time delay is $\theta=6 \mathrm{~h}$ and the the corresponding proportional gain is $\mathrm{Kp}=4.857$ $\mathrm{h}^{-1}$ and integral time $\tau_{1}=170 \mathrm{~h}$ for the closed-loop time constant $\lambda=35 \mathrm{~h}$ and the control's gain for the linearizing $\mathrm{I} / \mathrm{O}$ controller is $\tau=1.0 \mathrm{~h}^{-}{ }^{1}$, for the proposed controller the gains are selected as; $g_{1}=$ $-2.857 \mathrm{~h}^{-1}$ and $g_{2}=0.5 \mathrm{~g}^{-\mathrm{w}+1} \mathrm{~L}^{-\mathrm{w}+1} \mathrm{~h}^{-1}$, considering $w$ $=3$.

The "Integral Time-Weighted Squared Error" (ITSE) defined by (18) suggested by Ogunnaike and Ray (1994) is considered to measure the impact of the control error. ITSE provides the advantage of heavy penalization of large errors at long time; therefore, an adequate measure of resilience of the considered controllers.

$$
\text { ITSE }=\int_{0}^{\infty} t \varepsilon^{2} d t
$$

As can be observed in Figure 2, the proposed methodology acts almost immediately, leading the sulfate trajectory to the corresponding set points without overshots and settling times, with an adequate effort of the control as shown in Figure 3. Note that all the controllers act over reachable operating regions, which is feasible for a real implementation. Figure 4 shows the closed-loop behavior of the uncontrolled states (biomass and sulfide concentrations); as can be observed, they are stable trajectories; therefore, it can be concluded that the bioreactor is stabilizable.

Figure 5 is related to a 3D phase-portrait, including all the trajectories of the state variables. Note that the trajectories remain in a bounded region. Figure 6 shows a comparison of the performance of the proposed controller against other control structures. As can be observed, the linear PI controller provides the worst performance, even more, the performance index (ITSE) does not reach a steady state value. The ideal linearizing I/O controller provides a satisfactory performance as observed because this controller cancels the nonlinearities of the system and imposes a closedloop linear behavior, leading the corresponding trajectory to the selected set points in an asymptotic and exponential way; however, note that the performance of the proposed controller is the best in comparison with the other control schemes, without the need for a perfect plant model. Figure 6 is related to the closed-loop behavior of the corresponding concentration trajectories, under different initial conditions, it can be observed that all of them reach the corresponding closed-loop steady-state. Furthermore, additional numerical simulations were done in order to show the effect of the polynomial degree on the proposed control performance. As proved in Section 3, when the order of the polynomial contribution increases, the corresponding closed-loop performance is improved. Figure 7 is related to the sulfate closedloop trajectory under several polynomial degrees and, finally, Figure 8 shows the corresponding performance index, which is in agreement with the expected result. 


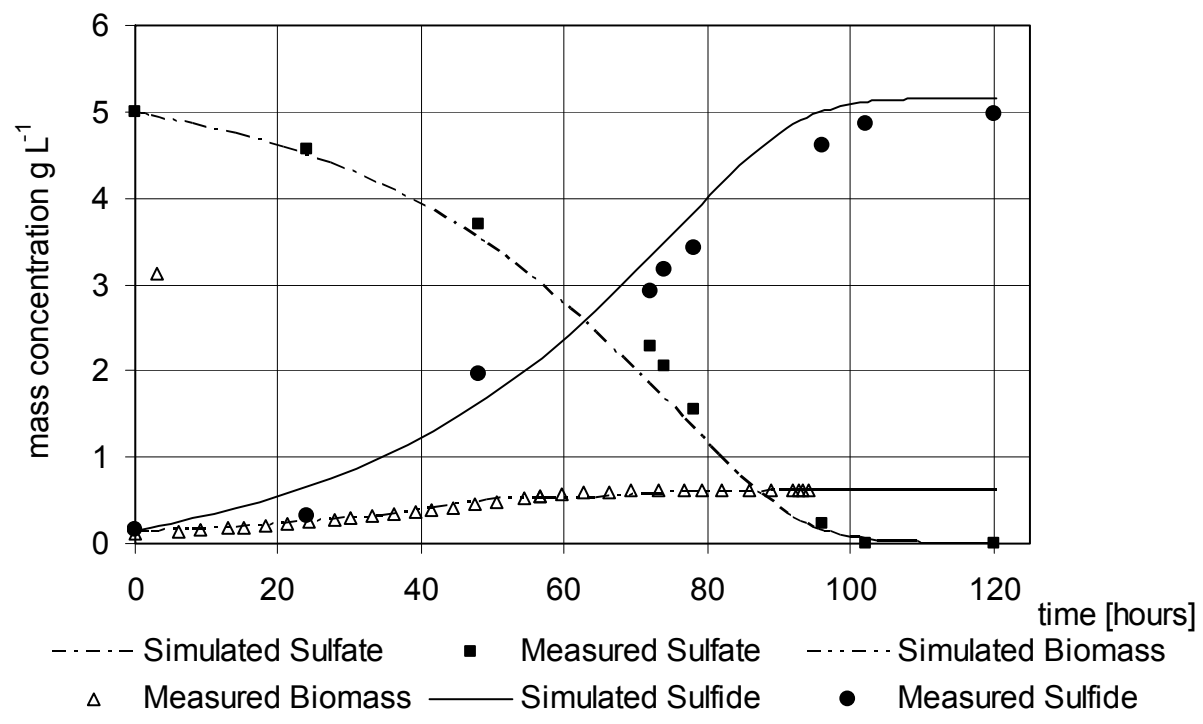

Figure 1. Experimental validation of the kinetic model.

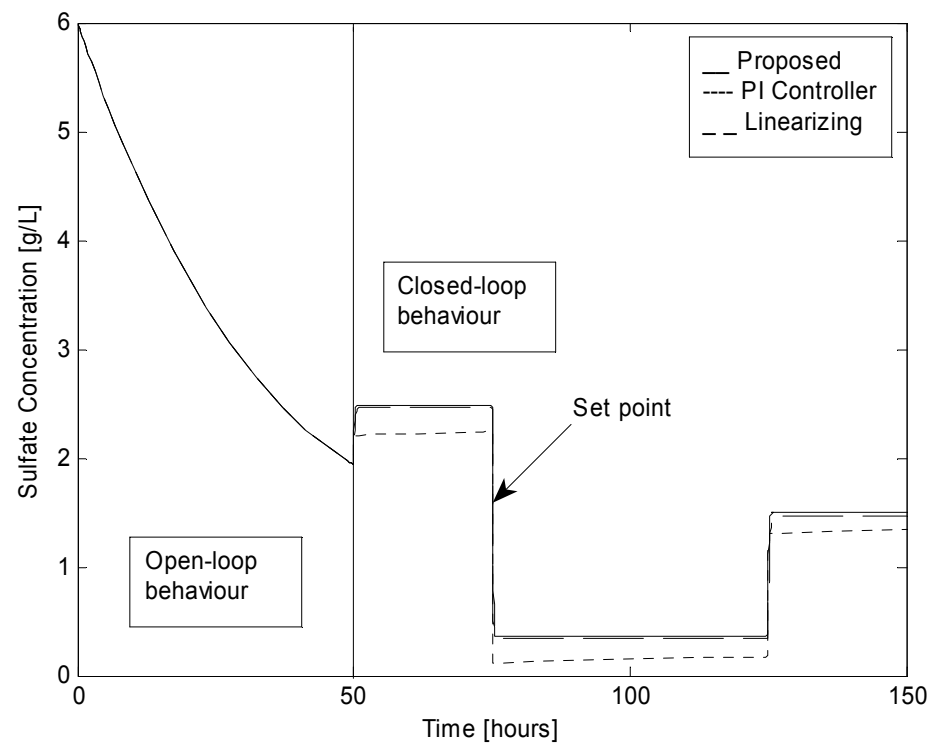

Figure 2. Closed-loop trajectory of the sulfate concentration. 


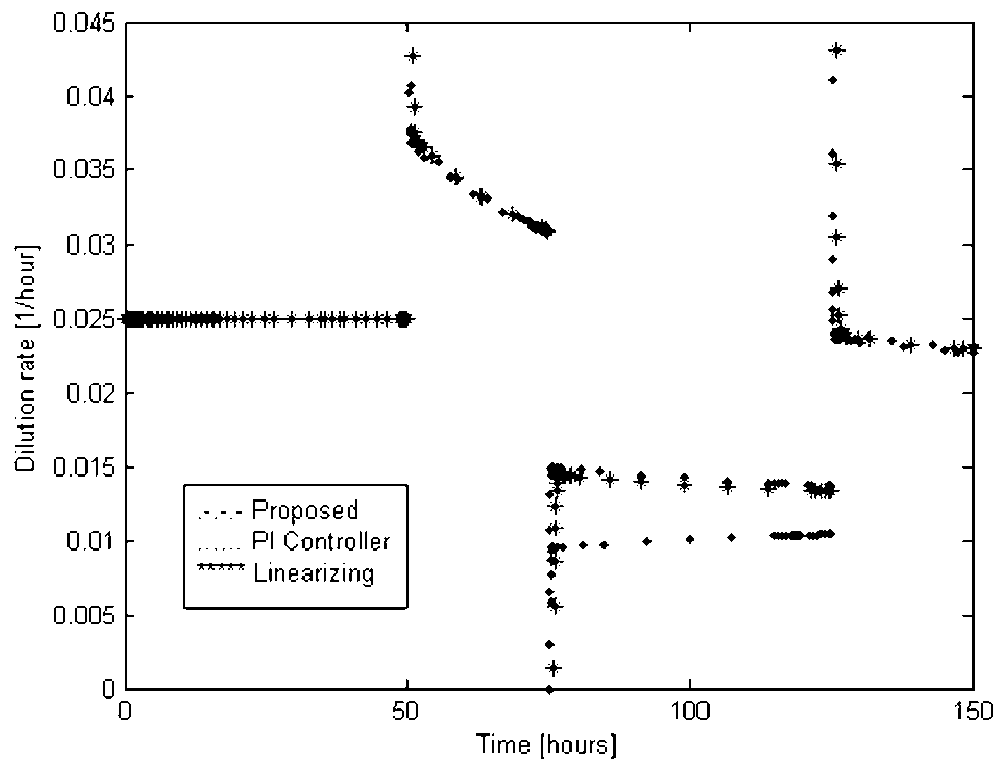

Figure 3. Control input efforts.

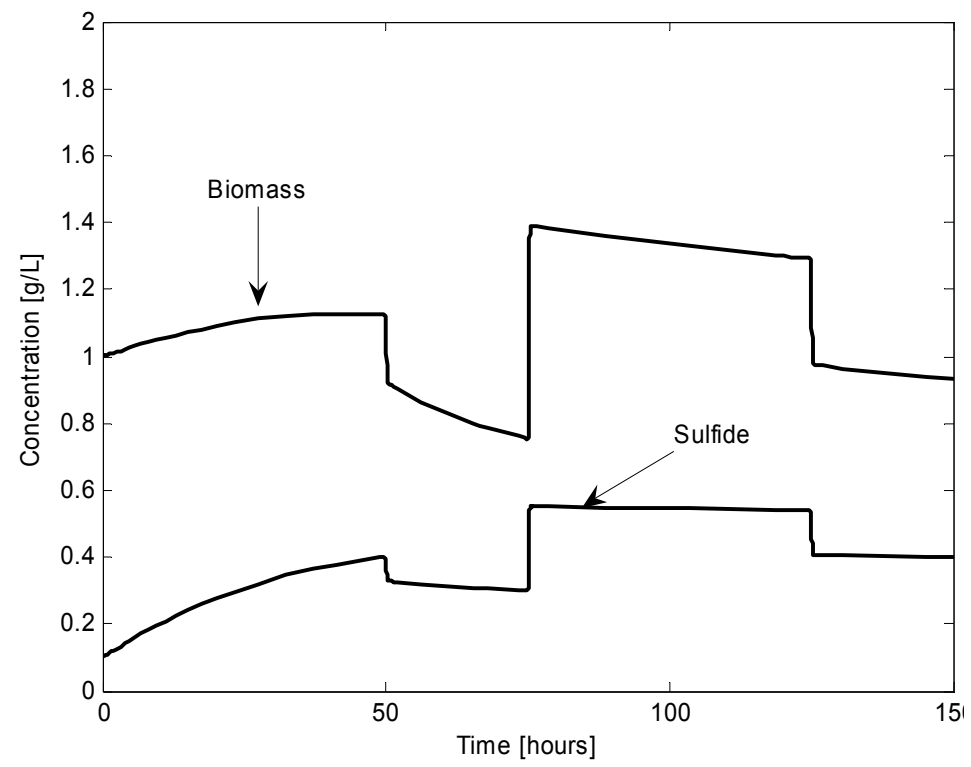

Figure 4. Closed-loop performance of the uncontrolled mass concentrations. 


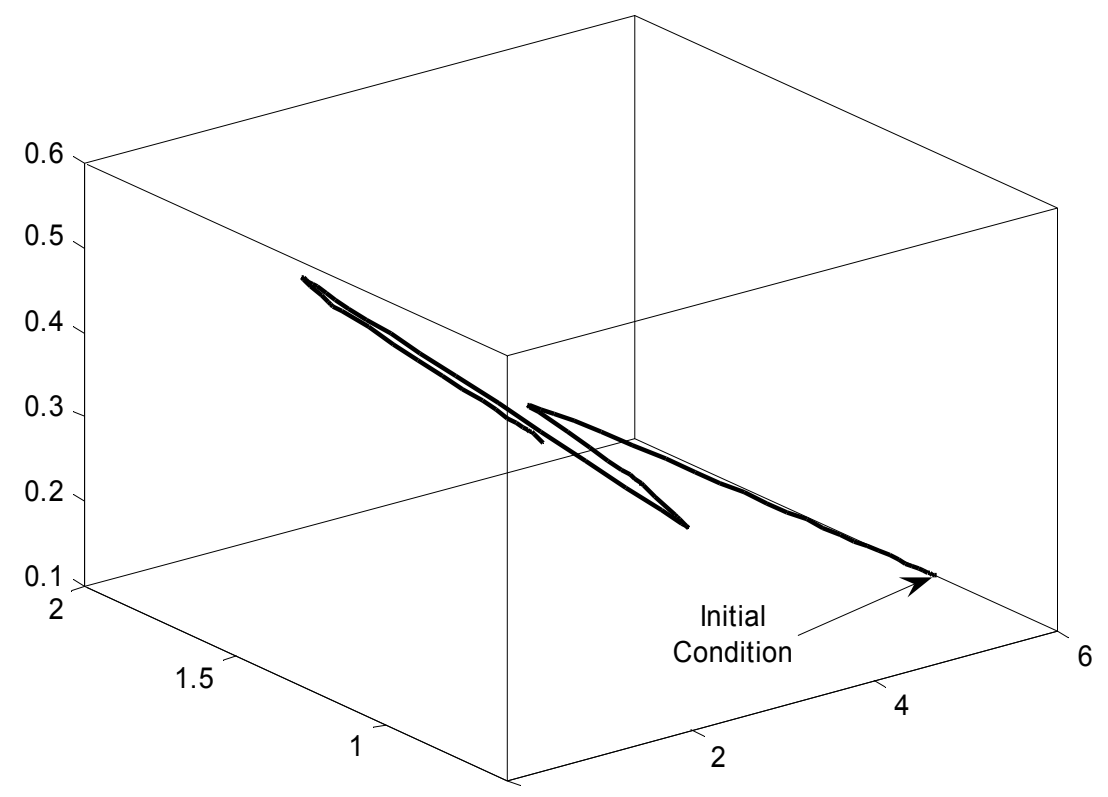

Figure 5. Closed-loop phase portrait.

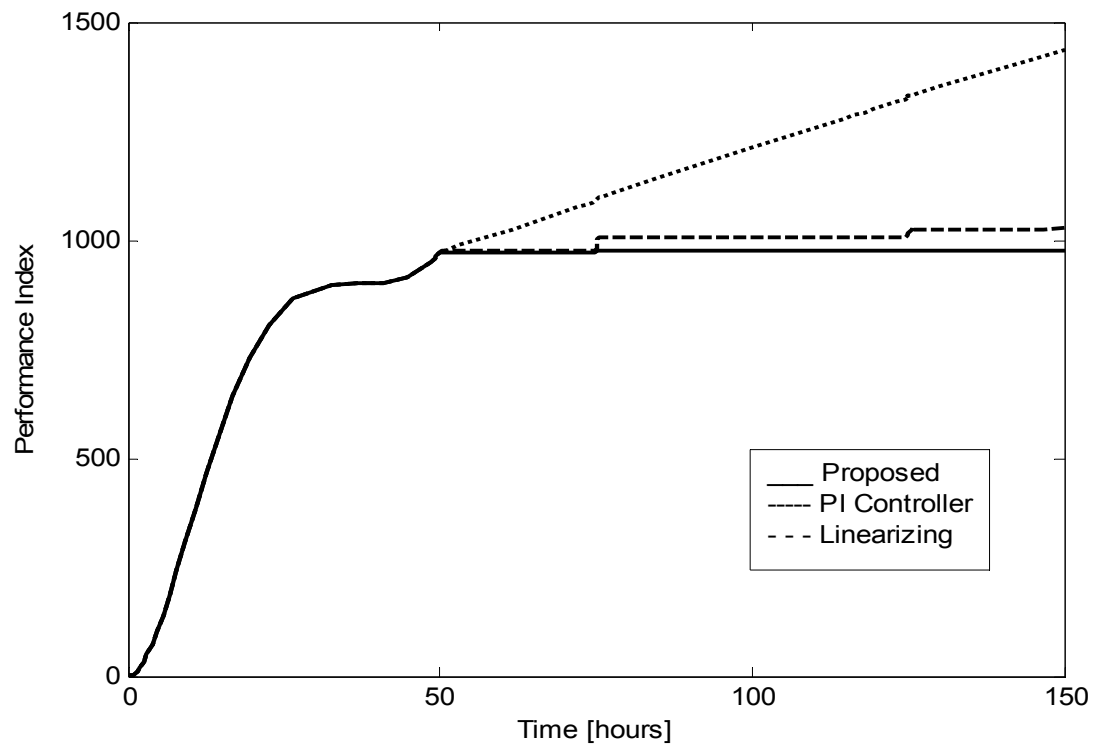

Figure 6. Performance index (ITSE) for the considered controllers. 


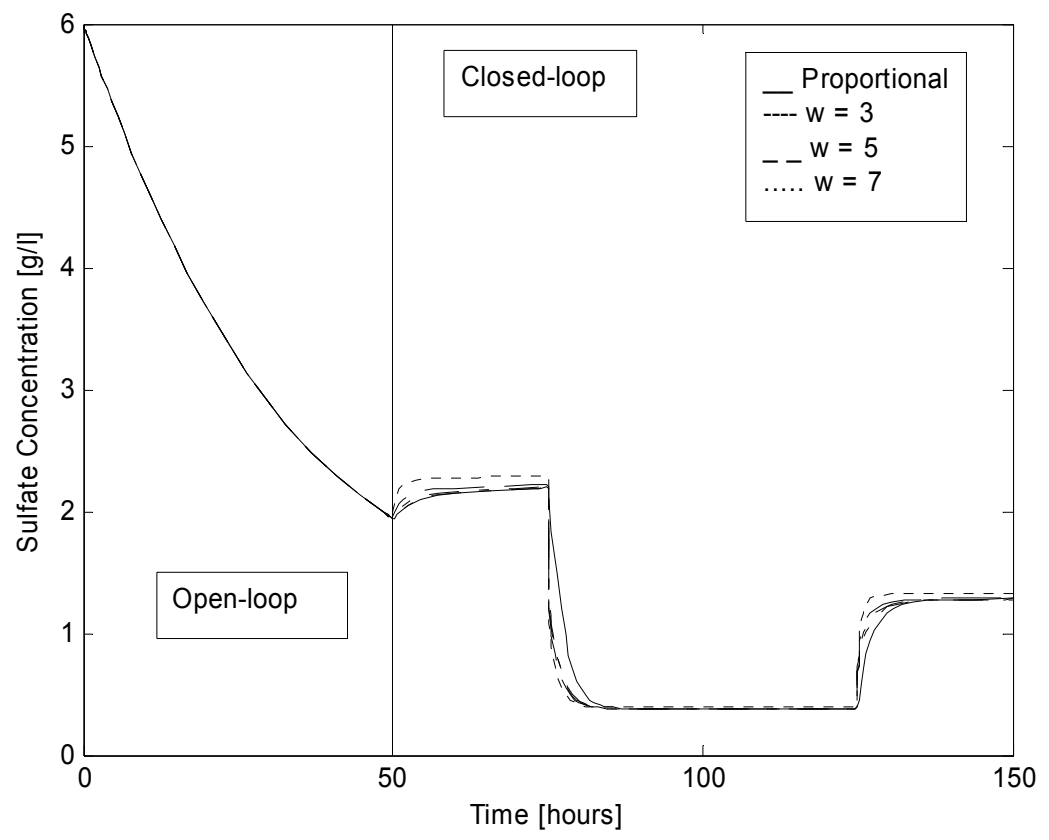

Figure 7. Closed-loop performance of the sulfate concentration for several values of the polynomial degree of the proposed controller.

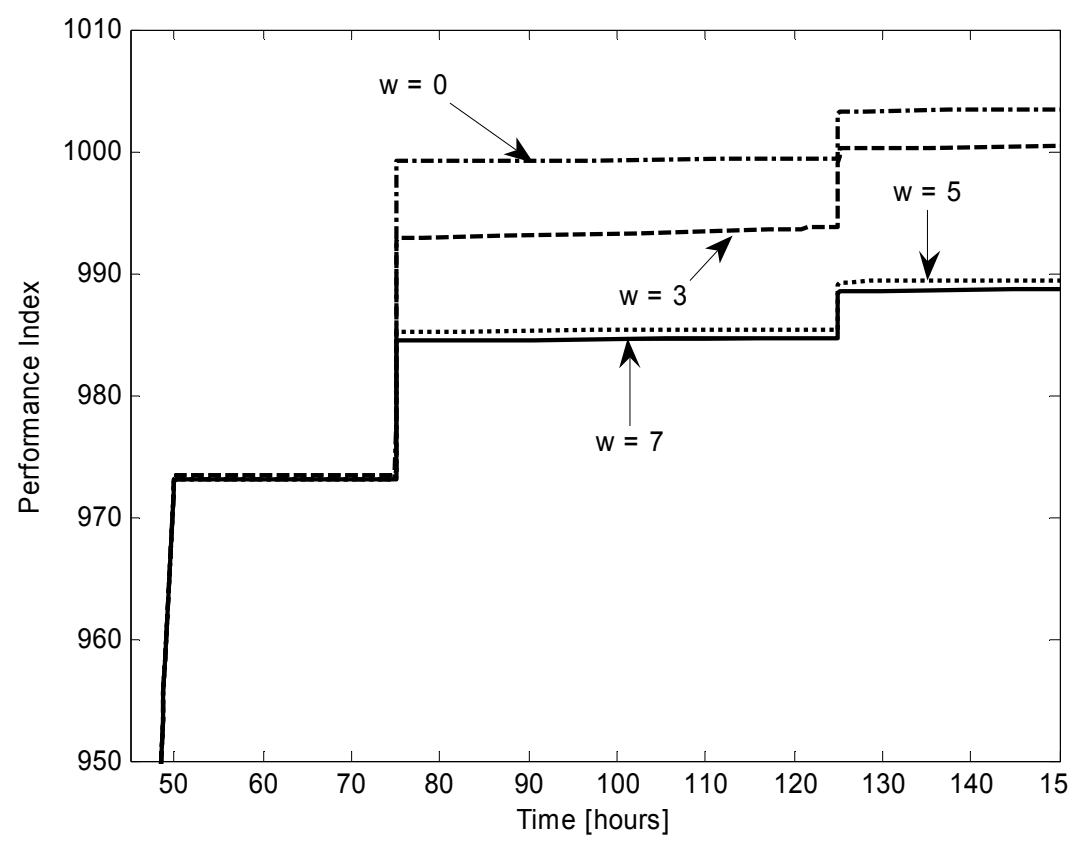

Figure 8. Performance index for several values of the polynomial degree of the proposed controller. 


\section{Concluding remarks}

In this paper, a class of nonlinear controller with polynomial output injection which provides semiglobal stabilization under the considered assumptions is proposed. The proposed controller is able to provide adequate performance for regulation and tracking purposes in a better way than a linearizing $\mathrm{I} / \mathrm{O}$ control law does and it is able to avoid overshots and setting time, in comparison with the linear PI control. As predicted, when the order of the polynomial increases, a better performance is achieved. No perfect knowledge of the model is required to design the controller which is a valuable feature process application.

\section{References}

[1] Alford JS, Bioprocess control: Advances and challenges. Computers and Chemical Engineering 30: 1464-1475 (2006).

[2] Aguilar R, Martínez SA, Rodríguez MG and Soto G, Process analysis for treatment of industrial plating wastewater: simulation and control approach. Chemical Engineering Journal 105: 139-145 (2005).

[3] Aguilar-López R, Neria-González MI, DomínguezBocanegra AR and Acevedo R, Robust generic model feedback under model uncertainties: Application to tubular reactor for treatment of industrial plating wastewater. Journal of Applied Research and Technology 3: 147-158 (2008).

[4] Baeza JA, Ferreira EC and Lafuente J, KnowledgeBased Supervision and Control of Wastewater Treatment Plant: a Real-Time Implementation, Water Science and Technology 12: 129-137 (2000).

[5] Bastin G and Dochain D, On-line Estimation and Adaptive Control of Bioreactors, Process Measurement and Control, Vol. 1. Elsevier: Amsterdam, (1990).

[6] Bailey JE and Ollis DF, Biochemical Engineering Fundamentals, Mc Graw Hill, Singapore (1986).

[7] Betancur MJ, Moreno JA, Moreno-Andrade I, Buitrón $\mathrm{G}$, Practical optimal control of fed-batch bioreactors for the waste water treatment. International Journal of Robust and Nonlinear Control 3: 173 - 190 (2006).

[8] Chidambaram M and See RPA, Simple method of tuning PID controllers for integrator/dead-time processes. Computer \& Chemical Engineering 27: 211215 (2002).

[9] Cord-Ruwisch R (1985) A quick method for determination of dissolved and precipitated sulfides in cultures of sulfate-reducing bacteria. J Microbiol Methods 4:33-6.

[10] Fordyce AP, Rawlings JB, Edgar TF, In Computer Control of Fermentation Processes D. Omstead, Eds. CRC Press, Inc., Florida, (1987).

Jorgensen BB, Mineralization of organic matter in a sea bed the role of sulfate reduction-, Nature 296: 643-645 (1982).

[11] Hungate RE. A roll tube method for cultivation of strict anaerobes In: Norris JR, Ribbons DW, editors. Methods in Microbiology, Vol. 3B. London: Academic Press; (1969) p 117-32.

[12] Kalafatis $A D$, Wang $L$ and Cluett WR, Linearizing feed forward-feedback control of $\mathrm{pH}$ process based on Wiener model. Journal of Process Control 15: 103 -112 (2005).

[13] Kolmert $A^{\circ}$, Wikstro" m P, Hallberg K (2000) A fast and simple turbidimetric method for the determination of sulfate in sulfate-reducing bacterial cultures. J. Microbiol. Meth. 41:179-184.

[14] Neria-González I, Wang ET, Ramírez V, Romero JM, Hernández-Rodríguez E (2006) Characterization of Bacterial Community Associated to Biofilms of Corroded Oil Pipelines from the Southeast of Mexico. Anaerobe 12: $122-133$.

[15] Neria.González MI and Aguilar-López R, Tracking Trajectories in a Continuous Anaerobic Bioreactor employing a Nonlinear Proportional Controller. Int. Journal of Chemical Reactor Engineering. 5 A73 (2007).

[16] Ogunnaike, B.A. \& Ray, W.H. Process, Dynamics, Modeling and Control. Oxford University Press. New York, 1994.

[17] Rivera ED, Morari M and Skogestad S, Internal model control 4. PID controller design. Industrial \& Engineering Chemistry Process Design and Development 1: 252-265 (1986).

[18] Schugerl K, Bioreaction Engineering, vol. 1. Wiley: Chichester (1987).

[19] Smets IYM, Versyck KJE and Van Impe JFM, Optimal control theory: a generic tool for identification and control of (bio-) chemical reactors. Annual Reviews in Control 26: 57-73 (2002). 
[20] Spear M, Time to turn up the speed? Process control developments continue to outrun their uptake by industry. Chemical Processing (2005), 23, 5, 345-349.

[21] Widdel $F$, In biology of anaerobic organism, ed Zehnder, AJB, Jhon Wiley, New York, pp 569-586 (1988).

[22] Ziegler JG and Nichols NB, Optimum settings for automatic controllers, Trans. ASME, 64: 759 - 765 (1942).

\section{Acknowledgments}

V.P.C. is very grateful with CONACyT for the support via a postgraduate scholarship. 


\section{Authors' Biographies}

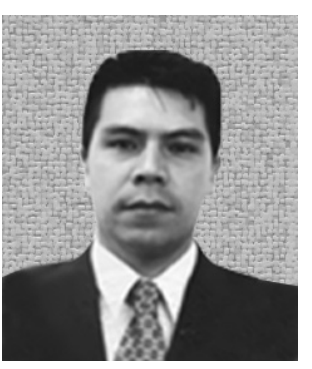

\section{Vicente PEÑA-CABALLERO}

He was born in Mexico City in 1973. He received a bachelor's degree in biotechnology engineering and an Msc degree in bioprocess from Unidad Profesional Interdisciplinaria de Biotecnología in 2001 and 2008, respectively. Nowadays, he is a Ph D student in the Departamento de Biotecnología y Bioingeniería at CINVESTAV-IPN, where he is working on modeling and control of continuous bioreactors. He is author of several papers published in national conferences proceedings.

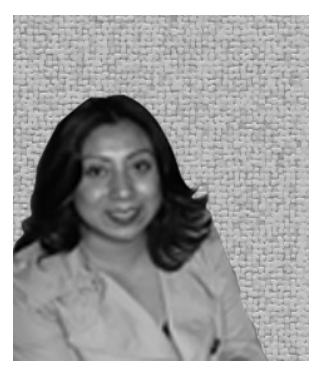

\section{María Isabel NERIA-GONZÁLEZ}

She was born in Mexico City in 1971. She is a biochemical engineer from Universidad Autónoma Metropolitana (1999), she ears her M sc and Ph D degrees in microbiology science from Escuela Nacional de Ciencias Biológicas, IPN (2002 and 2006, respectively). She was a postdoctoral fellow in the Departamento de Biotecnología y Bioingeniería at CINVESTAV-IPN and in the Microbiology Department of Provence University, France. She is author and/or coauthor of 16 published papers in indexed journals. Currently, she is a full professor at Tecnológico de Estudios Superiores de Ecatepec and candidate to become a member of the National System of Researchers (SNI).

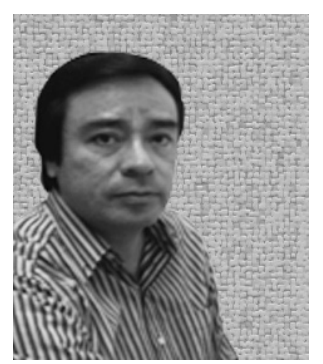

\section{Ricardo AGUILAR-LÓPEZ}

He was born in Mexico City in 1964. He received his B Sc, M Sc and PhD degrees from Universidad Autónoma Metropolitana in 1989, 1993 and 1998, respectively, all of them in chemical engineering. He was a postdoctoral fellow at Instituto Mexicano del Petróleo (2000); besides, he ears a PhD degree in automatic control from CINVESTAV-IPN (2003). He works on robust observer design for nonlinear systems and process control. He is author and co-author of 88 papers published in international journals. Currently, he is a researcher at the Departamento de Biotecnología y Bioingeniería of the Centro de Investigación y Estudios Avanzados, IPN. Dr. Aguilar has been a member of the National System of Researchers since 1998 (level II). 